\title{
PREMIUM CALCULATION: \\ WHY STANDARD DEVIATION SHOULD BE REPLACED BY ABSOLUTE DEVIATION ${ }^{1}$
}

\author{
By Dieter Denneberg
}

Bremen, FRG

\begin{abstract}
Average absolute (instead of quadratic) deviation from median (instead of expectation) is better suited to determine the safety loading for insurance premiums than standard deviation: The corresponding premium functionals behave additive under the practically relevant risk sharing schemes between first insurer and reinsurer.
\end{abstract}

\section{KEYWORDS}

Premium principles; average absolute deviation; comonotonic additivity; distorted probabilities.

\section{INTRODUCTION}

If one looks into the extensive literature on premium principles one gets the impression that actuaries are more or less incontent with the premium principles known till now. For example there was not known a nontrivial functional on nonnegative random variables, in actuarial terms a premium principle for insurance contracts, with the following elementary and plausible requirements: P1. The safety loading (premium minus expected value) is nonnegative, P2. no ripoff, i.e. the premium does not exceed the maximal claim, P3. consistency, i.e. the safety loading does not change if claims are augmented by a non-random constant and P4. proportionality, i.e. insuring a certain percentage of total damage costs that percentage of full insurance. It should be mentioned that the proportionality property $\mathrm{P} 4$ despite its practical importance is not regarded desirable by all authors (e.g. GERBER). We shall discuss that point at the end of section three.

The present article intends to make actuaries familiar with a broad class of functionals with properties P1 through P4. These functionals had been developed (by SchmeIDLeR, YAARI and others) during the last decade in the context of economic decision theory with the intention to overcome the

1 Lecture, given under the title "Quantilsabhängige Prämienprinzipien" at 21. Tagung der ASTIN-Gruppe in der DGVM, October 13th, 1989, Stuttgart. 
controversely discussed shortcomings of expected utility theory. Expected utility had been used, too, to construct premium functionals as the exponential principle, favoured e.g. by GERBER.

To make things as easy and accessible as possible we confine ourselves to an elementary one parameter class of premium functionals of the YAARI type (DENNEBERG 1985, 1988a and b). This functional resembles the standard deviation principle, where the safety loading is proportional to standard deviation. But the volatility measure standard deviation is replaced by average absolute deviation from the median and, surprisingly, all works.

In the first section we compile the properties of average absolute deviation from median, a volatility measure which nowadays is nearly forgotten, whereas in the first part of our century it enjoyed equal rights with standard deviation (e.g. in Czuber, cf. the discussion in Denneberg 1988b). The median being a quantile, it is appropriate here and in the sequel to employ the quantile function instead of its inverse function, the usual distribution function.

The premium functional with safety loading proportional to absolute deviation is introduced in the second section and properties P1 through P4 and some others-here we stress only subadditivity - are verified.

In section three the basic issue of comonotonicity of several random variables is introduced which, in some sense, is opposite to independence. Comonotonicity means that the risks involved are not able to compensate each other and this property implies additivity of our premiums. If risks are shared, e.g. between first insurer and reinsurer, the partial risks are comonotonic for most risk sharing schemes, among them all practically relevant ones. Hence our premium functional is compatible with the pratice of reinsurance. We discuss comonotonic additivity, a property not shared by the standard deviation principle, versus independence additivity, a property shared by the variance and exponential principles.

The final section gives an outlook on the more general class of premium functionals mentioned above. There is a further well known volatility measure, which, like absolute deviation, is associated to that class: the Gini coefficient. It might be interesting for pricing reinsurance.

\section{QUANTILE FUNCTION AND ABSOLUTE DEVIATION}

Let $X$ be a random variable to be interpreted as claims from an insurance contract or from a portfolio of such claims. We assume the increasing distribution function $F=F_{X}$ of $X$ to be known. $F(x), x \in \mathbb{R}$, denotes the probability of the event $X \leq x$. For our purposes the inverse function $\check{F}$ of $F$ is better suited to represent the distribution of $X$ than $F$. Since $F$, in general, is not one to one (e.g. for discrete distributions), we have to be cautious in defining $\breve{F}$. First, for $q$ in the unit interval $[0,1]$ we define the $q$-quantile of $X$ to be the interval $\left[\inf _{F(x) \geq q} x, \sup _{F(x) \leq q} x\right]$. The $\frac{1}{2}$-quantile is the median of $X$. For all $q \in[0,1]$ outside possibly a countable set the $q$-quantile of $X$ reduces to a single point. Now we define $\breve{F}(q)$ to be some fixed point of the $q$-quantile of $X$. 
Then $M X:=\check{F}\left(\frac{1}{2}\right)$ is a median of $X$. There is possibly an arbitrariness in the definition of $\breve{F}$ and $M X$ but this does not affect the values of the subsequent integrals. For short the function $\breve{F}$ will be called the quantile function of $X$.

The expectation of $X$ is

$$
E X=\int_{-\infty}^{\infty} x d F(x)=\int_{0}^{1} \check{F}(q) d q
$$

and we will make use of the absolute and quadratic norms

$$
\left\|\left.X\right|_{1}:=E|X|, \quad\right\| X \|_{2}:=\left(E\left(X^{2}\right)\right)^{1 / 2} .
$$

The corresponding volatility parameters are average absolute deviation from median $\tau=\tau(X)$ and standard deviation $\sigma=\sigma(X)$ :

$$
\tau:=\|X-M X\|_{1}, \quad \sigma=\|X-E X\|_{2} .
$$

It is natural to take the real numbers $M X$ and $E X$ as points of reference in defining the respective volatility parameter since these numbers minimise the respective distance from $X$ :

$$
\tau=\min _{a \in \mathbb{R}}\|X-a\|_{1}, \quad \sigma=\min _{a \in \mathbb{R}}\|X-a\|_{2} .
$$

If one looks for a parameter to indicate asymmetries of distributions one encounters two main methods. Either one uses higher odd moments, e.g. $E\left(x^{3}\right)$, or semivariances. The analogous to the latter in the case of absolute deviation are

$$
\tau_{-} \cdot=\int_{0}^{1 / 2}|\check{F}(q)-M X| d q, \quad \tau_{+}:=\int_{1 / 2}^{1}|\check{F}(q)-M X| d q
$$

and one has

$$
\begin{aligned}
\tau & =\tau_{+}+\tau_{-} \\
E X-M X & =\tau_{+}-\tau_{-} .
\end{aligned}
$$

From these equations we derive, that the triple $\left(M X, \tau_{-}, \tau_{+}\right)$of parameters contains the same information about the distribution of $X$ as the triple $(E X, M X, \tau)$.

Finally we prove subadditivity of $\tau$ and $\sigma$,

$$
\tau(X+Y) \leq \tau(X)+\tau(Y), \quad \sigma(X+Y) \leq \sigma(X)+\sigma(Y) .
$$

For standard deviation this is simply the triangle inequality for the norm $\|\cdot\|_{2}$. In case of absolute deviation, apart from the triangle inequality for the norm \|\|$_{1}$, one needs the above minimal property of the median to cope with the fact that the median is not additive: 


$$
\begin{aligned}
\tau(X+Y) & =\|X+Y-M(X+Y)\|_{1}=\min _{a \in \mathbb{R}}\|X+Y-a\|_{1} \leq\|X+Y-(M X+M Y)\|_{1} \\
& \leq\|X-M X\|_{1}+\|Y-M Y\|_{1}=\tau(X)+\tau(Y) .
\end{aligned}
$$

In section 3 there will be given a sufficient condition for additivity of $\tau$ analogous to additivity of variance $\sigma^{2}$ in case of independance.

\section{THE ABSOLUTE DEVIATION PRINCIPLE AND ELEMENTARY PROPERTIES}

Let $\mathfrak{X}$ be an appropriate set of random variables, e.g. the linear space $L^{1}$ or $L^{2}$ of random variables $X$ on a fixed probability space with finite norm $\|X\|_{1}$ and $\|X\|_{2}$, respectively. In our context, a functional

$$
H: \mathfrak{X} \rightarrow \mathbb{R}, \quad X \mapsto H X
$$

is called a premium functional or premium principle. The properties $P 1$ through $\mathbf{P 4}$ from the introduction read in formal terms

P1. $H X \geq E X$

P2. $H X \leq \sup X$

P3. $H(X+c)=H X+c, \quad c \in \mathbb{R}$

P4. $H(c X)=c H X, \quad c \geq 0$.

Under the premium principles, studied in actuarial literature till now, only the trivial functionals $H=E$ (net premium principle) and $H=\sup$ (maximal loss principle) have all four properties. The common standard deviation principle

$$
H X=E X+a \sigma(X), \quad X \in L^{2}, \quad \text { with parameter } a>0
$$

for example, violates $\mathrm{P} 2$. Our new premium functional

$$
H X:=E X+\rho \tau(X), \quad X \in L^{1}, \quad \text { with parameter } 0 \leq \rho \leq 1
$$

is constructed in the same way and will be called absolute deviation principle.

It is worth mentioning that this functional coincides with the expected value principle for special distributions: namely if $M X=0$ and $\tau_{-}(X)=0$, i.e. $X \geq 0$ and the probability of no claim is $\geq 1 / 2$. Then $H X=(1+\rho) E X$.

The absolute deviation principle can be expressed, too, by the three parameters median $M X$, average negative and positive deviation $\tau_{-}(X)$ and $\tau_{+}(X)$ from the median (see section 1):

$$
H X=M X-(1-\rho) \tau_{-}(X)+(1+\rho) \tau_{+}(X) .
$$

In this form the functional can be made plausible, too. The median serves as a reference point. Positive deviations, i.e. larger claims, are weighted more than negative deviations, i.e. smaller claims, and total weight is one.

We get an integral representation for the absolute deviation principle if, in the last formula, we replace $\tau_{-}$and $\tau_{+}$by their defining integrals : 


$$
\begin{aligned}
H X & =M X-(1-\rho) \int_{0}^{1 / 2}\left(M X-\breve{F}_{X}(q)\right) d q+(1+\rho) \int_{1 / 2}^{1}\left(\check{F}_{X}(q)-M X\right) d q \\
& =\int_{0}^{1 / 2} \check{F}_{X}(q)(1-\rho) d q+\int_{1 / 2}^{1} \check{F}_{X}(q)(1+\rho) d q .
\end{aligned}
$$

Let $\gamma$ denote the distribution function on the unit interval with density $1-\rho$ on $[0,1 / 2[$ and $1+\rho$ on $[1 / 2,1]$, then

Now we can prove the

$$
H X=\int_{0}^{1} \breve{F}_{X}(q) d \gamma(q)
$$

Theorem. The absolute deviation principle has properties P1 through P4 and

P5. $\check{F}_{X} \leq \check{F}_{Y}$ implies $H X \leq H Y$

P6. $H(X+Y) \leq H X+H Y$

P7. $H$ is (Lipschitz-) continuous on $L^{1}$ :

$$
|H X-H Y| \leq(1+\rho)\|X-Y\|_{1} .
$$

In P5 the condition $\breve{F}_{X} \leq \check{F}_{Y}$ (to be formally correct here, take e.g. right continuous quantile functions) is equivalent to $F_{X} \geq F_{Y}$ and this condition is often called first order stochastic dominance of $Y$ over $X$. Hence P5 states compatibility of $H$ with that stochastic order. $\mathrm{P} 2$ is the special case $Y \equiv \sup X$.

Property P6 states subadditivity of the functional $H$. In the next section we will give conditions under which additivity holds. In the general case a formula for the deviation $H(X+Y)-(H X+H Y)$ from additivity can be found in DENNEBERG 1985.

Proof of the theorem.

P1 is plain from the fact that $\rho \geq 0, \tau(X) \geq 0$.

$\mathrm{P} 2$ is, as we noted already, a special case of P5.

P3. $\breve{F}_{X+c}=\breve{F}_{X}+c$ and the assertion follows from the integral representation of $H$.

P4. For $c \geq 0$ one has $\breve{F}_{c X}=c \breve{F}_{X}$ (for negative $c$ the right hand side would no longer be an increasing function).

P5 is an immediate consequence of integral calculus.

P6 derives from additivity of expectation and subadditivity of $\tau$.

P7. $|H X-H Y|=\left|\int_{0}^{1}\left(\check{F}_{X}(q)-\check{F}_{Y}(q)\right) d \gamma(q)\right| \leq \int_{0}^{1}\left|\check{F}_{X}(q)-\check{F}_{Y}(q)\right| d \gamma(q)$

$$
\leq(1+\rho) \int_{0}^{1}\left|\breve{F}_{X}(q)-\breve{F}_{Y}(q)\right| d q \leq(1+\rho)\|X-Y\|_{1} .
$$


The last inequality is stated and proved as a separate lemma.

Lemma. For $X, Y \in L^{1}$ one has

$$
\left\|\breve{F}_{X}-\breve{F}_{Y}\right\|_{1} \leq\|X-Y\|_{1},
$$

where, on the left hand side, the norm refers to Lebesque measure on $[0,1]$.

Proof. Denote by $X \vee Y$ the maximum and by $X \wedge Y$ the minimum of the random variables $X, Y$. The inequalities

$$
X \wedge Y \leq X, Y \leq X \vee Y
$$

imply

$$
\begin{aligned}
& \check{F}_{X \wedge Y} \leq \check{F}_{X}, \check{F}_{Y} \leq \check{F}_{X \vee Y}, \\
& \left|\check{F}_{X}-\check{F}_{Y}\right| \leq \check{F}_{X \vee Y}-\check{F}_{X \wedge Y} .
\end{aligned}
$$

By integration we get

$$
\left\|\breve{F}_{X}-\breve{F}_{Y}\right\|_{1} \leq \int\left(\breve{F}_{X \vee Y}(q)-\breve{F}_{X \wedge Y}(q)\right) d q=E(X \vee Y-X \wedge Y)=E|X-Y|=\|X-Y\|_{1}
$$

\section{COMONOTONICITY AND REINSURANCE}

Here we tackle the question under what conditions on $X$ and $Y$ one has equality in P6, i.e. additivity of $H$. The condition is that $X, Y$ are comonotonic random variables (a term introduced by SchMEIDLER and YAARI), i.e. per definitionem that one of the following equivalent conditions hold:

(i) (No risk compensation) For each $\omega_{0}$ as point of reference the functions $f:=X-X\left(\omega_{0}\right)$ and $g:=Y-Y\left(\omega_{0}\right)$ don't have opposite signs, i.e. $|f+g|=|f|+|g|$.

(ii) $X=u(Z)$ and $Y=v(Z)$ for some $Z$ and (weakly) increasing functions $u$, $v$.

(iii) $X=u(X+Y)$ and $Y=v(X+Y)$ with continuous, increasing functions $u, v$ such that $u(z)+v(z)=z, z \in \mathbb{R}$.

These conditions and the proof of their equivalence (Satz 7 in DENNEBERG 1989) is valid for real functions $X, Y$, the distributions don't play any role. But distributions are essential in the following theorem (Satz 1 in DENNEBERG 1989):

Theorem. For comonotonic random variables $X, Y$ the quantile functions behave additive,

$$
\check{F}_{X+Y}=\check{F}_{X}+\check{F}_{Y}
$$

Applied to the absolute deviation principle $H$ we get

P8. $H(X+Y)=H X+H Y$ for comonotonic $X, Y \in L^{1}$. 
The proof of the theorem uses the fact that for increasing $u$ one has $\breve{F}_{u(X)}=u \supset \breve{F}_{X}$. The proof is easy if all distribution functions and the functions $u, v$ in (iii) are one to one.

Returning for a moment to the first section we, too, have

$$
\tau(X+Y)=\tau(X)+\tau(Y) \quad \text { for comonotonic } \quad X, Y \in L^{1} .
$$

Hence comonotonicity plays the same role for average absolute deviation $\tau$ as independence plays for variance $\sigma^{2}$. But notice that independence and comonotonicity are opposite, mutual exclusive properties (except the case where $X$ or $Y$ is constant).

We give typical examples for comonotonic random variables.

Example. $u(x)=x^{+}:=\max \{0, x\}$ and $v(x)=-x^{-}$, where $x^{-}:=(-x)^{+}$, are continuous increasing functions and $u(x)+v(x)=x$. Hence, for a random variable $X$, the random variables $X^{+}=u(X)$ and $-X^{-}=v(X)$ are comonotonic. If $X$ has median $M X=0$ (this can be achieved by a translation) comonotonicity implies $\tau(X)=\tau\left(X^{+}\right)+\tau\left(X^{-}\right)$. This equation is known from Section 1 since $\tau\left(X^{+}\right)=\tau_{+}(X)$ and $\tau\left(-X^{-}\right)=\tau\left(X^{-}\right)=\tau_{-}(X)$ in case $M X=0$.

Example (excess of loss or stop loss reinsurance). Let $Z$ be total claims and $a$ the priority or stop loss point. Define $v(z):=(z-a)^{+}, u(z):=z-v(z)$ and $X:=u(Z), Y:=v(Z)$. Then $X$ is the part of total claims $Z=X+Y$ to be covered by the primary insurer and $Y$ the part to be covered by the reinsurer. $X, Y$ being comonotonic $H$ is compatible with this type of reinsurance, $H[Z]=H[X]+H[Y]$

We know already from $\mathrm{P} 4$ that $H$ is compatible, too, with proportional reinsurance. But we can state more. Condition (ii) or (iii) for comonotonicity in connection with $\mathrm{P} 8$ says that $H$ is compatible with very general risk sharing schemes. One has only the restriction that both risk sharing partners have to bear (weakly) more if total claims are higher. There are forms of reinsurance of minor or lacking practical importance which injure this condition and which are not compatible with $H$. An example is largest claims reinsurance.

The essential properties of our new premium functional have been derived now, and before looking on possible generalisations, we will discuss the crucial properties: proportionality $\mathrm{P} 4$, subadditivity $\mathrm{P} 6$ and comonotonic additivity P8. First notice that P4 can be derived from P8 using P5 or norm continuity. We will compare P6 and P8 with independence additivity. For the discussion it is essential to specify the situation in which a premium functional is to be applied. We distinguish two situations.

If the market for insurance is in equilibrium in the sense that it offers no arbitrage opportunity, prices are additive at least for independent risks. Thus 
premium functionals which are additive on independent risks, e.g. the variance principle $E X+a \sigma^{2}(X)$ and the exponential principle $\frac{1}{a} \ln E e^{a X}$, are candidates for modeling market prices.

On the other hand, subadditive but not additive premium functionals as our absolute deviation principle or the standard deviation principle are apt to depict the law of large numbers. Hence they are applicable in portfolio decisions. Here reinsurance is an important mean, may it be to reduce the ratio of the portfolios volatility to the companies equity below a desired limit, or may it be to reduce volatility of the various companies portfolios through risk exchanges such that, eventually, the companies portfolios become proportional to the market portfolio. In such decisions comonotonic additivity P8 which-as pointed out above - applies to most risk sharing schemes, is very useful and can simplify decisions. Notice that the standard deviation principle is not comonotonic additive.

\section{GENERALISATIONS AND THE GINI PRINCIPLE}

As the reader may have guessed already, the representation

$$
H X=\int_{0}^{1} \check{F}_{X}(q) d \gamma(q)
$$

of the absolute deviation principle is capable of generalisation. One can replace the piecewise linear function $\gamma$ by any distribution function on the unit interval $[0,1]$. Such a function $\gamma$ is called a distortion of probabilities. Condition PI means that the graph of $\gamma$ lies below the diagonal, $\gamma(q) \leq q$. P6 is valid if $\gamma$ is convex and has bounded density. For P7 bounded density is needed, too, and the Lipschitz constant is the supremum $\left\|\gamma^{\prime}\right\|_{\infty}$ of the density $\gamma^{\prime}\left(\left\|\gamma^{\prime}\right\|_{\infty}=1+\rho\right.$ in case of the absolute deviation principle). All the other properties remain valid without further restrictions. In DenNeberg 1989 (see also DenNeberg 1990) these assertions are proved and the converse, too: any functional $H$ on $L^{1}$ with properties P1 through P8 can be represented by the above integral with a convex distribution function $\gamma$ having bounded density.

Sometimes the absolute deviation principle may not be appropriate owing to the piecewise linearity of $\gamma$. For excess of loss or stop loss reinsurance the latter implies that the safety loading factor remains constant with rising priority or stop loss point, respectively. In practice one rather observes rising safety loading factors, too. Already the next simple distortion allows to model this phenomenon. For the absolute deviation principle the density can be written as

$$
\gamma^{\prime}(q)=1+\rho \operatorname{sgn}\left(q-\frac{1}{2}\right) .
$$


Replace the signum by the next elementary odd function, the identity:

$$
\gamma^{\prime}(q)=1+\rho\left(q-\frac{1}{2}\right)
$$

The corresponding distortion is the quadratic polynomial $\gamma(q)=q+\frac{1}{2} \rho\left(q^{2}-q\right)$, which is convex on $[0,1]$ for $0 \leq \rho \leq 2$, and the premium functional is

$$
H X=E X+\rho \frac{1}{2} \text { Gini } X
$$

where

$$
\text { Gini } X:=\int_{0}^{1} \breve{F}(q) d q^{2}-E X=E X \text { gini } X
$$

and gini $X$ is the (normed) Gini coefficient, which is used in economic welfare theory as an inequality measure for wealth distributions in populations. The usual definition for the Gini coefficient is twice the area between the diagnoal and the Lorenz function

$$
\begin{aligned}
& l(q):=\frac{1}{E X} \int_{0}^{q} \check{F}(p) d p, \\
& \text { gini } X=2 \int_{0}^{1}(q-l(q)) d q .
\end{aligned}
$$

The equivalence to the above formula is calculated easily with Fubinis theorem. Another representation of the Gini coefficient is

$$
\text { Gini } X=\frac{1}{2}\|X-Y\|_{1}
$$

where $Y$ is a random variable such that $X, Y$ are independent and identically distributed (see ZAGIER). This new premium functional could be called the Gini principle.

The above general premium functional can further be generalised. First the basic probability measure $P$ or the distorted $\gamma \circ P$ can be replaced by more general set functions. Second-as in expected utility - the claims in money terms can be valued by a non linear utility function. Thus the proportionality property P4 could be weakened. Functionals of this type and their axiomatic representations are investigated in economic decision theory (see e.g. WAKKER, where the literature is discussed, too). 


\section{REFERENCES}

Czuber, E. (1903, 3. Aufl. 1913, 1. Band) Wahrscheinlichkeitsrechnung und ihre Anwendung auf Fehlerausgleichung, Statistik und Lebensversicherung. Teubner, Leipzig und Berlin.

Denneberg, D. (1985) Valuation of First Moment Risk for Decision Purposes in Finance and Insurance. In H. GöPl, R. HENN (Hrg.) 3. Tagung Geld, Banken und Versicherungen, Karlsruhe 12.-15.12.1984. Karlsruhe (Verlag Versicherungswirtschaft), 855-869.

Denneberg, D. (1988a) Keynes Decision Rule, on 'Expectation' in the General Theory. European Journal of Political Economy 4, 205-223.

DenneberG, D. (1988b) Risiko und Erwartung in der Allgemeinen Theory, eine Formalisierung. In H. Hagemann, O. Steiger (ed.'s) Keynes' General Theory nach fünfzig Jahren. Berlin : Duncker \& Humblot.

DeNNeBERG, D. (1989) Verzerrte Wahrscheinlichkeiten in der Versicherungsmathematik, quantilsabhängige Prämienprinzipien. Mathematik-Arbeitspapiere Nr. 34 Preprint, FB Mathematik/Informatik, Universität Bremen.

Denneberg, D. (1990) Distorted Probabilities and Insurance Premiums (Extended Abstract of Denneberg 1989). In Proceedings of 14. SOR, Ulm Sept. 6-8, 1989. Frankfurt (Athenäum).

GERBER, H.U. (1979) An Introduction to Mathematical Risk Theory. S. S. Huebner Foundation Monograph Series No. 8. Homewodd/III (Irwin).

SCHMEIDLER, D. (1986) Integral Representation without Additivity. Proceedings of the American Mathematical Society 97, 255-261.

Wakker, Peter P. (1989) Additive Representations of Preferences, a New Foundation of Decision Analysis. Theory and Decision Library Series C. Dordrecht (Kluwer).

YAARI, M.E. (1987) The Dual Theory of Choice under Risk. Econometrica 55, 95-115.

ZAGIER, D. (1983) Inequalities for the Gini Coefficient of composite populations. Journal of Mathematical Economics 12, 103-118.

\section{DenneberG}

FB Mathematik/Informatik, Universität Bremen, D-2800 Bremen 33. 